\title{
SISTEMA DE GESTIÓN INTELIGENTE PARA SUMINISTROS DE AGUA Y ENERGÍA ELÉCTRICA EN UN EDIFICIO MILITAR BASADO EN EL INTERNET DE LAS COSAS.
}

\author{
${ }^{1}$ Cardenas Chavez Juan Carlos Mdol, ${ }^{1}$ Raymundo Roque Elkhy Berenice, ${ }^{1}$ Alan \\ Carrasco Valderrama
}

${ }^{1}$ Centro De Investigación Y Desarrollo De Proyectos, Fuerza Aérea Del Perú. Lima, Perú

Recibido: 05/09/2021 Revisado: 03/10/2021 Aceptado: 16/11/2021 Publicado: 30/01/2022

\section{RESUMEN}

Con el incremento de las tarifas de los suministros de agua y energía eléctrica, el tema del inadecuado uso de recursos en un edificio militar por parte de los usuarios ha recibido mucha atención.

Con el objetivo de monitorear el entorno de las instalaciones del edificio militar, controlar los aparatos eléctricos para reducir el consumo de energía, controlar el dispensado de agua, y analizar el entorno y la utilización en la instalación, este documento desarrolla el sistema de gestión inteligente para suministros de agua y energía eléctrica en un edificio militar basado en el internet de las cosas.

En este sistema, el conjunto de sensores tales como: módulos sensores para Arduino y tarjetas de sensores personalizadas, como también el conjunto de actuadores tales como: módulos actuadores para Arduino y tarjetas de actuadores se utiliza como conjunto básico del internet de las cosas.

El modelo de almacenamiento lo establece la base de datos relacional MYSQL. Además de la aplicación móvil que servirá de interfaz de usuario.

Los gastos de agua y energía eléctrica en el edificio militar se reducen hasta cierto punto y el desaprovechamiento de recursos en el edificio militar se reduce satisfactoriamente.

\section{ABSTRACT}

With the increase in tariffs for water and electricity supplies, the issue of inappropriate use of resources in a military building by users has received much attention.

In order to monitor the environment of the military building facilities, control electrical appliances to reduce energy consumption, control water dispensing, and analyze the environment and use in the facility, this document develops the intelligent management 
system for water and electrical power supplies in a military building based on the internet of things.

In this system, the sensor assembly such as: Arduino sensor modules and custom sensor cards, as well as the actuator assembly such as: Arduino actuator modules and actuator cards are used as the basic assembly of the Internet of Things.

The storage model is established by the MYSQL relational database. In addition to the mobile application that will serve as the user interface.

The expenses of water and electric power in the military building are reduced to a certain extent, and the waste of resources in the military building is reduced satisfactorily.

\section{PALABRAS CLAVES}

IOT Internet of things / Internet de las cosas

APP Aplication movile / Aplicación móvil

ARDUINO Open-source electronic prototyping platform / plataforma prototipado electrónico abierto

REDES Redes informáticas

SENSORES Dispositivos sensibles a magnitudes

MYSQL Administrador Base de datos

\section{INTRODUCCIÓN}

Debido al considerable crecimiento de las tarifas de suministros de agua y energía eléctrica, consecuencia de los acuerdos entre generadores y distribuidores de energía, y por presentarse una disminución de las reservas de agua elevando los costos de producción. Además del inadecuado aprovechamiento de estos recursos por parte de los usuarios. Estos dos aspectos dan como consecuencia un incremento en el gasto de los suministros de agua y energía eléctrica tanto como para los hogares, fábricas, edificios empresariales y en nuestro caso los edificios militares.

Debido a la poca promoción sobre el uso consciente de los recursos como el agua y la energía eléctrica, se puede observar casos donde se desaprovecha el agua, electrodomésticos y equipos informáticos conectados innecesariamente e iluminación en ambientes cuando no es necesario.

Sin embargo, en el proceso de control automático, es necesario evaluar una cantidad significativa de parámetros, como automáticamente apagar las luces de la instalación, y es necesario evaluar la intensidad de luz externa en ese momento para así proceder o no. Al 
mismo tiempo, el entorno interior tiene un impacto directo en la salud, la calidad de vida y la eficiencia en el trabajo.

Con el desarrollo del internet de las cosas, las personas pueden obtener el entorno circundante a un costo menor. Para lograr el ahorro de recursos en el edificio militar, es necesario un control inteligente de lámparas, aires acondicionados, equipos informáticos, electrodomésticos y control de dispensado de agua. Sin embargo, para no afectar el ambiente de trabajo de los usuarios del edificio militar, es necesario encontrar un equilibrio que permita el ahorro de los recursos, reducir los costos operativos y satisfacer las necesidades de los usuarios.

Por tanto, se necesitan datos ambientales de las instalaciones para registrar las actividades de los usuarios, los cambios ambientales y el uso de los recursos, y analizar los datos en la etapa posterior para encontrar un punto de equilibrio. Sin embargo, la cantidad de datos generados espontáneamente por el internet de las cosas es grande. Cada instalación genera una cantidad significativa de datos ambientales todos los días. La base de datos relacional debe cumplir teniendo en cuenta esas demandas.

Con respecto a la conectividad en las instalaciones es necesario recurrir al acceso inalámbrico y por cable proporcionan una experiencia en línea estable, confiable y limitada. Sobre esta base, es factible configurar puertas de enlace en las instalaciones y oficinas para cargar datos ambientales y aceptar comandos para controlar los dispositivos interiores.

Con el aumento de dispositivos, la dirección ip del dispositivo de puerta de enlace y la identificación del sensor de origen pueden causar errores de almacenamiento. Siendo necesario un eficiente sistema de gestión de datos mediante un esquema de almacenamiento y lectura confiable para administrar los datos.

\section{Hipótesis:}

El desarrollo del proyecto de gestión inteligente de energia, que se instalará en los Edificios de la Fuerza Aérea del Perú, permitirá un ahorro energético, económico y generar conciencia en el uso de los recursos energéticos que en un futuro puedan acabarse.

\section{Objetivo de la investigación}

El desarrollo del proyecto de gestión inteligente de energia, tiene como objetivo el ahorro y reducción del gasto energético de los edificios de la Fuerza Aérea del Perú, además de generar conciencia en los usuarios sobre el uso eficiente de los recursos.

\section{Antecedentes del problema}

El cambio climático y el agotamiento de los recursos implican la eficiencia energética o eléctrica como un asunto social clave. Dado que representan el $40 \%$ del consumo total 
de energía, la eficiencia energética en los edificios constituye un potencial considerable de ahorro de energía.

La eficiencia energética, eficiencia eléctrica o eficiencia de la energía sirve para ahorrar dinero ya que solo usamos la energía que necesitamos. Pero también sirve para cuidar el medio ambiente. Podemos dar muchos ejemplos de eficiencia energética, entre ellos: En el hogar cuando usamos sensores o detectores de movimiento o presencia para apagar la luz cuando no haya personas. O usando equipos electrodomésticos como refrigeradoras, televisores, cocinas, entre otros que tiene un tipo de eficiencia energética.

\section{Importancia}

Mediante el desarrollo de un sistema de gestión inteligente de energia se podrá reducir el gasto de energia y promover un consumo eficiente de la energia de modo que el excedente se pueda usar inicialmente para financiar el sistema y para futuro cubrir otras necesidades.

Aplicación del proyecto para la FAP, la industrial o el País.

Para reducir el gasto energético y económico de los edificios de la Fuerza Aérea del Perú.

Para reducir el gasto energético, mejorar los procesos y hacer un eficiente consumo de los empleados.

Para el país la posibilidad de mantener la producción energética para mas años sin tener problemas de escases.

\section{METODOLOGÍA}

Para este proyecto de investigación consideramos tres (03) métodos principales:

\section{Método 1:}

Se pretende implementar el sistema para seis (06) instalaciones del edificio militar para ello se aplicará el método de Agile Testing destinada para la generación de productos modulo por modulo. De esta manera poder construir los módulos sensores, módulos actuadores y dispositivos de puerta de enlace para luego interconectarse e integrarse a una red a un mini servidor asociado a una base de datos. 


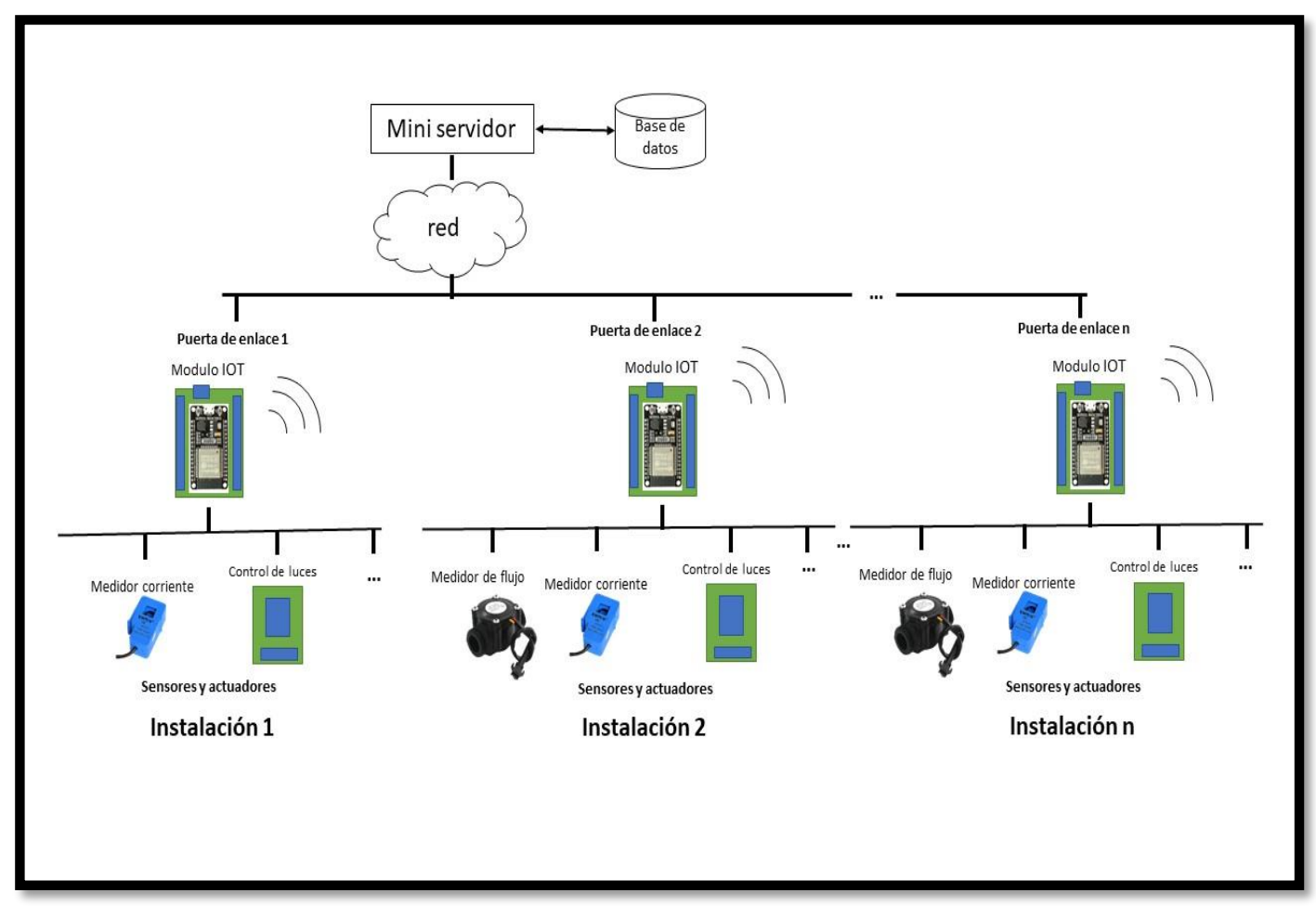

BIOTECH \& ENGINEERING Untels. Ene -Jun.2(1), 2022; ISSN:2788 -4295; 336-344 


\section{Método 2:}

Para el desarrollo de la aplicación móvil se utilizará el método extreme programming, que es una metodología ágil avocada a promover las relaciones interpersonales para conseguir objeto en el desarrollo de aplicaciones. el trabajo en equipo y el intercambio de conocimientos. Esta aplicación servirá para realizar el monitoreo y control del sistema. Esta metodología también nos servirá para la gestión de la base de datos (MySQ1) con el mini servidor (raspberry pi 4).

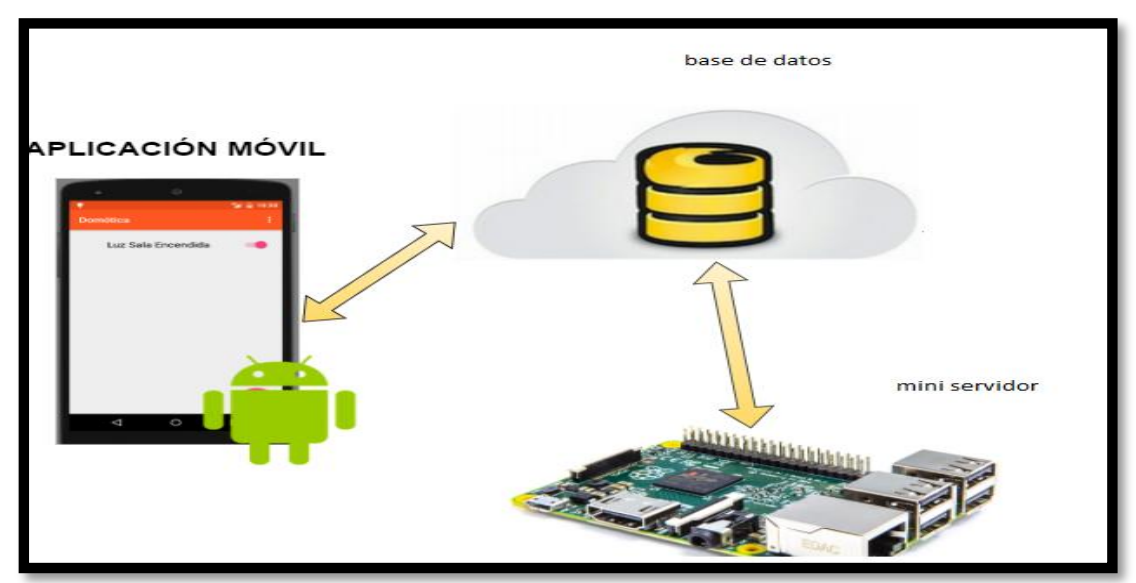

Método 3:

Para la recopilación y estudio de la información se utilizará un estudio de casos. El propósito de este desarrollo del sistema es recopilar datos ambientales de las instalaciones del edificio militar, analizar el uso de la instalación, y así reducir el consumo energético de las instalaciones. Para ellos se realizará la instalación del sistema a las 6 instalaciones donde se destinará 2 meses para las pruebas y luego se realizará la recopilación de datos por un mes.

Toda esta recopilación de datos se almacenará en una base de datos basado en un modelo relacional, lo cual nos ayuda en primera instancia para el almacenamiento como también para realizar las respectivas consultas.

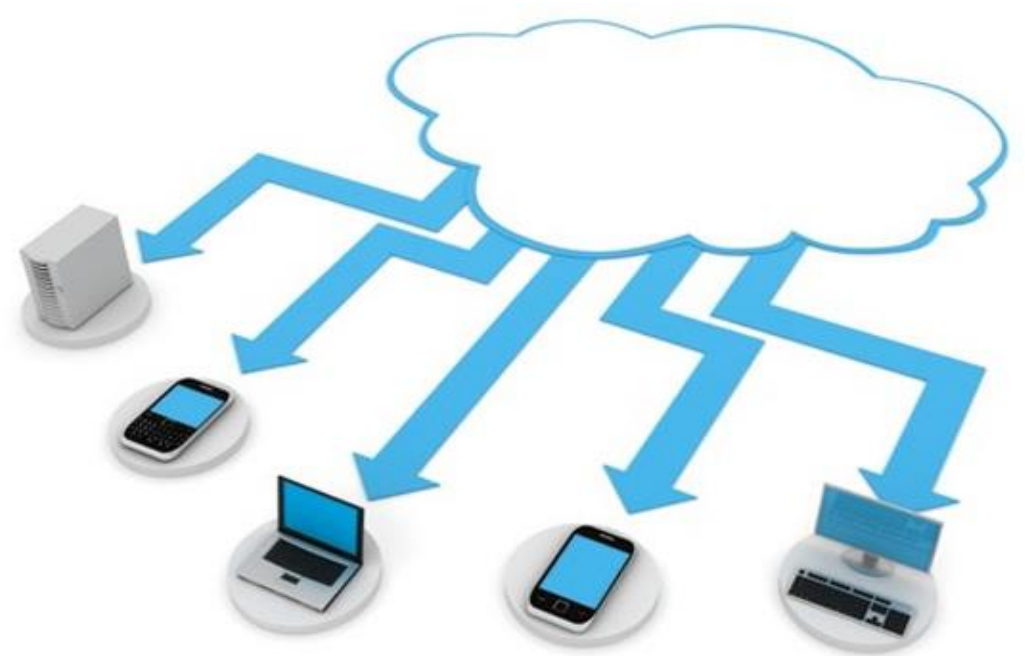

BIOTECH \& ENGINEERING Untels. Ene -Jun.2(1), 2022; ISSN:2788 -4295; 336-344 


\section{RESULTADOS}

Los resultados esperados para nuestro proyecto de investigación son conseguir una reducción del gasto de los servicios de agua y energía eléctrica de un $20 \%$.

Se espera una reducción del consumo de los electrodomésticos y equipos informáticos de un $20 \%$.

Se espera una reducción del consumo del agua en un $10 \%$.

\section{CONCLUSIONES}

Es posible conseguir una reducción del gasto de suministros de agua y energía eléctrica de manera considerable.

La tecnología del internet de las cosas brinda una nueva alternativa para el desarrollo de sistema de gestión inteligente para diversas aplicaciones.

El desarrollo de mejores módulos de IOT con mayores velocidades de procesamiento y medios de conectividad logran facilitar la recopilación de datos.

Los módulos sensores en la actualidad tiene una gran calidad y confiabilidad lo cual favorece a la correcta adquisición de datos.

El uso de base de datos mediante un modelo relacional para el almacenamiento de los datos es la mejor alternativa para el análisis de los datos.

El uso de metodologías agiles favorece mucho para acortar los tiempos en el desarrollo de proyectos y en la elaboración de prototipos.

\section{REFERENCIAS}

Peláez. J (2019). Internet de las cosas IoT con Arduino. Manual práctico. Paraninfo.

Velazco. E y Rey. J (2020). Diseño y gestión de edificios de consumo de energía casi nulo. Paraninfo.

Stone. R. (2019). Raspberry Pi 4 Manual Completo: Una guía paso a paso a la nueva Raspberry Pi 4 y a la creación de proyectos innovadores. Babelcube Inc.

Gilfillan. I (2018). La biblia de MySQL. Anaya. 


\section{ANEXO FOTOGRÁFICO}

\section{DIAGRAMA DE INSTALACION CONTROL LUCES}

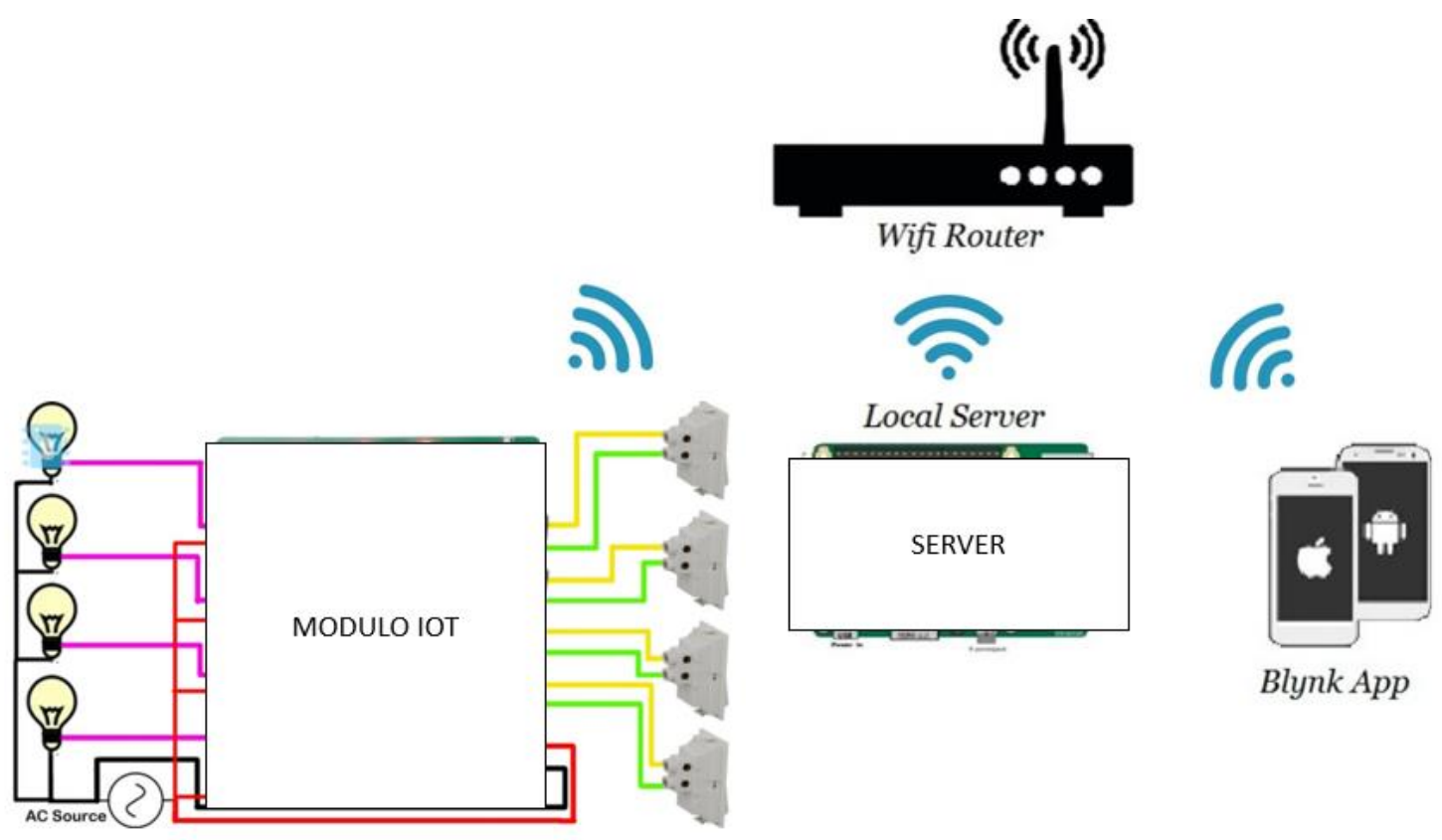


ถ)

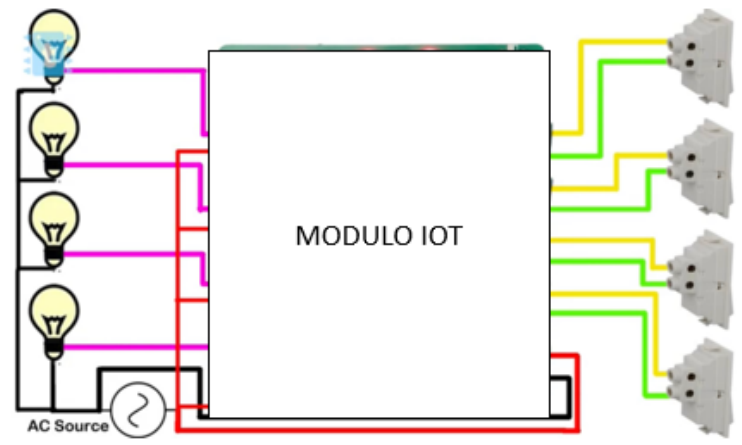

B)

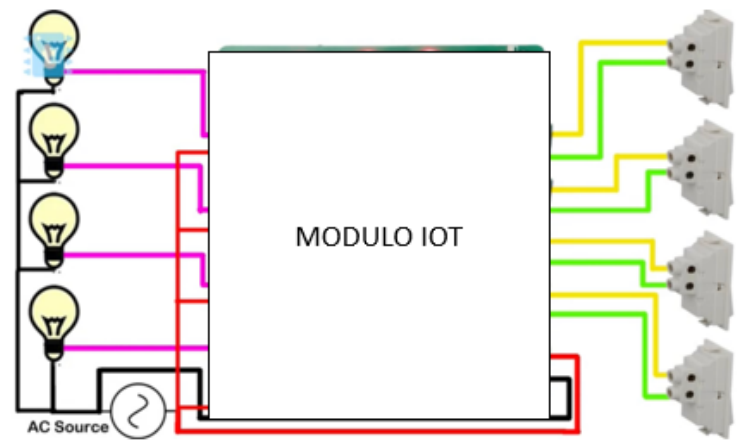

